\title{
LA RAZIONALITÀ APERTA DI OCKHAM ${ }^{*}$
}

Francesco Bottin ${ }^{* \star}$

SÍNTESE - São inumeras as interpretações que os modernos däo à obra de Ockham. No presente artigo, o autor afirma que Ockham é o primeiro pensador a conceber a explicação filosófica não mais como uma série de explicações concatenadas, a partir de princípios aceitos, mas como uma estratégia racional, que permite aos filósofos evitar uma série de problemas lingüisticos e conceituais.
ABSTRACT - The interpretations modernly given to Ockham's work are almost countless. In this paper the author asserts that Ockham is the first thinker conceiving the philosophical explication not more as a series of concatenated explanations, departing from accepted principles, but as a rational strategy, so that philosophers can avoid a great number of linguistic and conceptual problems.

\section{1- Distruzione, ricostruzione o anticipazione?}

L'operazione culturale compiuta da Ockham è apparsa agli studiosi ora come una forma di scetticismo che ha frantumato il tradizionale sapere metafisico (Michalski, de Lagarde), ora come un sovvertimento delle certezze (Giacon, Vasoli), ora come una catalisi di istanze critiche presenti nel suo tempo (Gilson). Altri vi hanno visto una anticipazione delle tendenze del pensiero logico e scientifico contemporaneo (Russell, Neurath, Moody, Panaccio), oppure hanno parlato di metamorfosi del discorso medievale (Leff); c'è stato chi ha caratterizzato il suo pensiero come una semiologia filosofica (de Andres). ${ }^{1}$ Certamente altre designazioni conti-

- Una precedente versione di questo saggio dal titolo, Ockhams offene Rationalität, è stato pubblicato in Die Gegenwart Ockhams, herausgegeben von W. Vossenkhul und R. Schönberger, VCH, Acta humaniora, Weinheim 1990, p. 51-62.

* Università di Padova.

1 Cfr. G. de LAGARDE, La naissance de l'esprit laique, vol. IV: Guillaume d'Ockham: défense de l'empire, Editions E. Nauwelaerts-Beatrice, Louvain-Paris 1962; K. MICHALSKI, La philosophie au XIVe siecle. Six etudes, herausgegeben von K. Flash, Minerva, Frankfurt am Main 1969; C. GIACON, Guglielmo di Ockham. Saggio storico-critico sulla formazione e sulla decadenza della Scolastica, Vita e Pensiero, Milano 1941; C. VASOLI, Guglielmo d'Occam, La Nuova Italia, Firenze 1953; E. GILSON, La filosofia nel Medioevo. Dalle origini patristiche alla fine del XIV secolo, La Nuova Italia, Firenze 1973, p. 787: "... il terminismo di Ockham non è stato la sola origine della crisi dottrinale degli anni 1339-1347, benchè, per la sua stessa natura, la sua dottrina abbia giocato naturalmente, per dir cosi, il ruolo di un catalizzatore"; B. RUSSELL, Storia della filosofia occidentale, Milano 1966; O. NEURATH, Il Circolo di Vienna e l'avvenire dell'empinismo logico, introd. di F. Barone, Borla, Roma 1977,

\begin{tabular}{|l|l|l|l|l|l|}
\hline VERTTAS & Porto Alegre & v. 41 & $\mathrm{n}^{0} 163$ & Setembro 1996 & p. 473-482 \\
\hline
\end{tabular}


nueranno ancora a venire proposte sulla base di fondate motivazioni. Ovviamente molte di queste caratterizzazioni del passato, come pure quelle più recenti e quelle che continuano a venire proposte, possono coesistere e gli storici del pensiero continueranno giustamente a riequilibrare una figura che è stata troppo facilmente sottoposta a fraintendimenti. La documentazione storica e la maggior conoscenza delle sue opere costituiscono il terreno sul quale tali modificazioni permettono di fondare via via l'immagine filosofica che ci si può fare di lui. Una volta eliminati i più gravi fraintendimenti del suo pensiero, una volta evidenziato questo o quell'aspetto delle sue dottrine sulla base di letture piủ attente, resta pur tuttavia una domanda alla quale lo storico non può rispondere da solo: qual è il vero senso della operazione culturale di questo pensatore nei confronti dei molti altri pensatori che affollano un periodo eccezionalmente vivace di quella stagione medievale?

\section{2 - Una strategia razionale}

Se i documenti storici e dottrinali stabiliscono i parametri entro i quali è lecito cercare una risposta, questa non sarà esaustiva se si limiterà a riproporre tali documenti senza illuminarli di epoca in epoca con la luce intellettuale propria di ogni età. La nostra età ha già dimostrato ampiamente di avere un feeling tutto particolare verso Guglielmo di Ockham giustificato dalla vicinanza con questa o quella dottrina contemporanea; ora, però, siamo forse in grado di capire in termini più generali da che cosa esso dipende. A mio avviso è indagando l'atteggiamento intellettuale di Ockham nei riguardi della spiegazione razionale che si può cogliere l'elemento più caratteristico del suo pensiero e nello stesso tempo il punto che lo rende a noi più vicino. È mia intenzione sostenere che Ockham è il primo pensatore a concepire la spiegazione filosofica non più come una serie di dimostrazioni concatenate a partire da principi accettati, ma come una strategia razionale tale da consentirci di sfuggire agli intoppi linguistici e concettuali che i filosofi incontrano immancabilmente nel loro cammino. Sono convinto che sia possibile analizzare le sue dottrine, ad iniziare da quella preliminare della sua critica alle entità astratte, come esemplificazioni di una strategia razionale da mettere in atto di volta in volta, piuttosto che come determinazioni definitive di un pensiero colto nella sua apoditticità teoretica.

\section{1 - II "rasoio di Ockham": principio filosofico o fiat metodologico?}

Il passo preliminare verso questo atteggiamento è stato certamente compiuto da Ockham con la elaborazione del suo cosiddetto rasoiò, sulla cui natura pure si registrano le oscillazioni interpretative più diverse che poi si ripercuotono su tutto il suo pensiero: esso è stato visto come una critica pseudo-metafisica alla metafisica, come una disamina logico-linguistica, come un criterio gnoseologico generale.

p. 67: "Russell, in Inghilterra, è in un certo senso il continuatore del nominalista Occam, il quale a Oxford affilava il suo strumento di analisi, il suo rasoio"; E. MOODY, The Logic of William of Ockham, Russell and Russell, New York 1965; C. PANACCIO, Les mots, les concepts et les choses. La sémantique de Guillaume d'Occam et le nominalisme d'aujourd'hui, Bellarmin-Vrin, Montréal-Paris 1991; G. LEFF, William of Ockham. The Metamorphosis of Scholastic Discourse, Manchester University Press, Manchester 1975; T. de ANDRES, El nominalismo de Guillermo de Ockham como filosofía del lenguaje, Gredos, Madrid 1969. 
Anche in questo caso le differenti caratterizzazioni possono, almeno in parte, coesistere, per quanto appaiano a prima vista in netta contrapposizione, se si intende "il rasoio di Ockham" non come un principio, ma come una condizione indispensabile che rende possibile concepire l'attività filosofica come una strategia razionale applicabile nei vari settori del sapere, dalla metafisica alla gnoseologia, dalla elaborazione di dottrine scientifiche all'etica. Non sembra che Ockham lo abbia mai utilizzato come un principio. D'altra parte, se fosse un principio, sarebbe un ben strano principio. Nessun principio che noi conosciamo è formulato in modo tale che la seconda parte della sua enunciazione "senza necessità" (sine necessitate) renda pressochè inutile la prima parte, "non si devono moltiplicare gli enti" (non sunt multiplicanda entia). Se preso troppo alla lettera potrebbe apparire simile da molti punti di vista al principio che non si danno eccezioni, salvo nei casi eccezionali.

Giustamente Gàl ha osservato che probabilmente Ockham sarebbe stato disposto ad accettare anche l'anti-rasoio che Chatton gli opponeva; "se non sono sufficienti tre cose per stabilire la verità di un enunciato affermativo, si deve postularne un quarto, e cosi via" ${ }^{2}$ come l'altra faccia della stessa moneta. Di sicuro Ockham non rifiutò mai l'anti-rasoio in quanto tale, ma pretese che esso fosse correttamente inteso. ${ }^{3}$ La difficoltà di definire questo presunto "principio" che gli interpreti contemporanei incontrano è la prova più chiara che per Ockham esso non doveva essere inteso come un principio. Esso ha piuttosto l'aspetto di un fiat metodologico basato su una convenzione comunemente accettata, relativa alla natura delle spiegazioni filosofiche. A mio avviso, per Ockham nè il suo rasoio, nè l'anti-rasoio di Chatton dovevano apparire come principi filosofici in contrasto con i principi sostenuti da altri (tuttavia Chatton lo dichiarava orgogliosamente regula mea) ${ }^{4}$ e soprattutto esso doveva venire utilizzato per aprire la discussione e l'argomentazione razionale, non per bloccarla in un'altra strettoia, diversa da quella criticata, ma pur sempre una strettoia che limitava l'uso degli strumenti razionali di dimostrazione.

\section{2 - Una astuzia logica e metodologica?}

Da questo punto di vista questo assunto della economia concettuale, ben noto anche a molti altri pensatori, nelle mani di Ockham, diventa assai spesso un'astuzia filosofica, lecita perchè da tutti accettata o quanto meno non rifiutabile in quanto tale, per far uscire la discussione dalle rotaie linguistiche e concettuali imposte dai vari filosofi. Ciò che è importante non è l'esatto significato che esso può

2 Cfr. G. Gàl in Introductio a OCKHAM, Summa logicae, p. 61*: "si tres res non sufficiunt ad verificandam propositionem affirmativam, oportet ponere quartam, et sic deinceps". I riferimenti alle opere di Ockham sono desunti sempre dall'edizione del Franciscan Institute, St. Bonaventure, New York.

Cfr. OCKHAM, Quodlibeta I, q. 5 : "... quando propositio verificatur pro rebus, si duae res non sufficiunt ad verificandam propositionem, oportet ponere tertiam ...; (p. 31, 40-42) ... dico quod principium in quo fundatur ista ratio falsum est, nisi melius intelligatur; quia ad veritatem alicuius propositionis quandoque sufficiunt duae res uno tempore, quandoque non sufficiunt duae res, puta alio tempore" (p. 32, 72). Per una ampia discussione dei rapporti tra il rasoio di Ockham e l'anti-rasoio di Chatton si veda. A. MAURER, Ockham's Razor and Chatton's Anti-razor, "Mediaeval Studies", 46(1984), p. 463-475.

Cfr. Gàl, Introductio, p. 61*. 
assumere nelle diverse situazioni nelle quali viene applicato, ma il fatto che esso, comunque, preceda qualsiasi principio, in qualsiasi disciplina. Ovviamente, ciò non significa che i principi perdono il loro valore, ma significa semplicemente che anch'essi vengono considerati validi come stratagemmi razionali per certi scopi. I principi posti come assoluti all'inizio della spiegazione, senza un adeguato accordo sulla natura di tale spiegazione, finiscono per determinare l'utilizzazione degli strumenti razionali in nostro possesso in un solco già predisposto. L'unica via di uscita in tal caso è quella di contrapporre principi a principi, visione a visione; diventa di fatto impossibile l'interazione e il confronto delle posizioni, diventa impossibile una vera ed aperta argomentazione razionale. Lo scopo della filosofia è quello di capire i modi in cui la razionalità si sviluppa, invece che quello di fissarne i caratteri una volta per tutte.

\section{3 - Le due radices degli errori filosofici}

Il dibattito filosofico vero e proprio inizia ovviamente dimostrando, di volta in volta, che certi filosofi hanno fatto uso di realtà non necessarie per presentare una spiegazione filosofica esauriente che può essere ottenuta anche senza tali entità. $\mathrm{Ma}$ fare ciò è una cosa molto differente dal proporre semplicemente una convenzione metodologica. Era necessario fare uso di una strategia dimostrativa convincente. Ockham, pertanto, individua due ambiti entro i quali sviluppare la sua strategia razionale nelle discussioni filosofiche : un ambito linguistico e un ambito più chiaramente ontologico. In questi due ambiti si annidano le due radices degli errori filosofici.

La prima radix dipende dal fatto che "taluni si basano esclusivamente sul significato letterale della filosofia greca tradotta in latino, che ha invece dato a molti occasione di errore" cioè logicamente e filologicamente inaccettabile assumere troppo alla lettera i termini della filosofia greca tradotti in latino. ${ }^{5}$ Egli, quindi non ha dubbi nell'imporre la propria interpretazione di Aristotele non su basi filologiche (per quanto lamenti le pessime traduzioni e le conseguenti difficoltà concettuali), ma su basi di coerenza logica. Una volta stabilita la complessità della filosofia di Aristotele nei punti più discussi ed accertato che l'assunzione della littera del testo

5 Cfr. OCKHAM, Summa logicae, p. 170, 232-171, 239: "nonnulli nimis innituntur proprietati sermonis vulgatae philosophiae, quae multis praebuit occasionem erroris". Ockham spiega questa radix facendo esplicitamente riferimento alla defectuosa translatio delle opere di Aristotele: "... tum quia defectuose translata; tum quia propter obscuritatem Graeci sermonis in Latinum translati male intelligitur et perversus inde quandoque intellectus elicitur; tum quia dicta auctorum falso saepissime allegantur, cum tamen etiam veraciter allegatis fidem non expediat adhibere multorum errorum late respersis". In base a questa personale convinzione, non ulteriormente specificata a livello filologico, Ockham è convinto che la filosofia aristotelica, cosi' come è conosciuta in traduzione latina, "... potest multis modis intelligi" (De motu, p. 316, 19). Egli, pertanto, non esita a correggere il modo comune di intendere le dottrine aristoteliche ritenendo di pervenire in tal modo al vero pensiero di Aristotele: "... istam [propositionem] intendit Philosophus, quamvis aliam ponat, sicut frequenter ponit unam propositionem pro alia" (In lib. Phys., IV-VIII, vol. V, p. 209, 21-25). Osservazioni analoghe vengono poroposte da Ockham ogniqualvolta è costretto a discostarsi dall'interpretazione comune dei testi aristotelici. Un interessante risultato al quale conduce questo metodo interpretativo è fornito da L.M. de Rijk al riguardo della concezione occamiana della dimostrazione: cfr. L. M. de RUK, Ockham's Theory of Demonstration: His Use of Aristotle's kath' holou and kath'hauto Requirements, in Die Gegenwart Ockhams, hsgg. von W. Vossenkhul und R. Schönberger, VCH, Weinheim 1990, p. 232-240. 
aristotelico non poteva costituire un criterio sufficiente di validità interpretativa, per il semplice fatto che le traduzioni risultavano quanto meno approssimative, Ockham sviluppa a pieno titolo la sua interpretazione che è basata sulle leggi universali della logica anzichè sulle mutevoli e contrastanti opinioni dei commentatori. Questa interpretazione, nella concezione di Ockham, si adatta perfettamente a tutto il pensiero dello Stagirita; basterà unicamente intervenire per precisare il genuino senso delle espressioni utilizzate dal filosofo greco, il quale, per svariati motivi, "non sempre parla in maniera esatta ... usando con rigore il significato dei termini".

La seconda radix consiste nel "moltiplicare gli enti in base al numero delle parole, in modo che si pretende che ad ogni parola corrisponda una cosa". ${ }^{7}$ Pertanto l'uso della logica e la disamina delle assunzioni ontologiche costituiscono il terreno specifico dell'impegno filosofico vero e proprio. Ed è su questo terreno che avviene lo scontro tra posizioni filosofiche differenti, non tanto sull'assunto metodologico in quanto tale. Tutti erano sempre stati, più o meno consapevolmente, favorevoli all'accettazione di tale assunto semplicemente perch esso sembra concernere la natura della spiegazione filosofica e non la legittimità delle singole assunzioni. Ogni spiegazione per essere tale deve certamente (i) essere in grado di connettere i dati di fatto accettati, ma (ii) nel postulare un legame tra entità apparentemente non correlate non deve creare problemi, logici e/o ontologici più gravi di quelli che intende risolvere. In altre parole, una spiegazione deve ridurre i gaps cognitivi e non semplicemente spostarli, magari aggravandoli. Naturalmente, singolarmente presi, nessuno dei filosofi riteneva di aver introdotto nel proprio sistema filosofico anelli esplicativi che risolvendo un problema ne aggravavano un altro, ma il contrasto a tutti evidente tra le varie posizioni filosofiche restava a documentare che la spiegazione era stata ottenuta generando nuovi e più gravi problemi. Soltanto quando Ockham metterà in atto la sua strategia critica su questi errori gli avversari si opporranno pensando di modificare anche l'assunzione metodologica iniziale con la elaborazione di un contro-rasoio. Ma ad Ockham esso non potrà che apparire come l'altra faccia della stessa assunzione.

\section{4- Rivoltare il sacco senza disperdere la farina}

Pertanto, egli potrà letteralmente "rivoltare il sacco della farina aristotelica", nel momento stesso in cui fa continuamente appello ai testi di Aristotele come a testi autorevoli proprio perchè si trattava di interpretare facendo uso di una logica adeguata le dottrine del filosofo greco e non di restare schiavi di termini o di presunti principi aristotelici. Alcuni contemporanei restarono fortemente irritati dalla sua

6 Crr. OCKHAM, Expositio in lib. Physicorum Aristotelis, lib. I, cap. 1, p. 28, 221: "non semper loquitur ... propriissime et de virtute sermonis ...".

7 Cfr. OCKHAM, Summa logicae, p. 171, 240-241 : "multiplicare entia secundum multitudinem terminorum et quod quilibet terminus habet quid rei". Si veda anche quest'altro passo della Summula philosophiae naturalis: "Et hoc est principium multorum errorum in philosophia: velle quod semper distincto vocabulo correspondeat distinctum significatum, ita quod tanta sit distinctio rerum significatarum quanta est nominum vel vocabolorum significantium, etiam quando illa nomina vel vocabula non sunt synonima. Sicut si aliquis crederet quod alia res significaretur per casum rectum et obliquum et per numerum singularem et pluralem, et per coniunctiones praepositiones et adverbia; quae sunt absurda et contra philosophiam" (p. 270, 87-94). 
operazione: Giovanni Luterell scriverà scandalizzato che Ockham stava sconvolgendo i principi fondamentali noti e accettati da tutti, anche dai ragazzi delle scuole. ${ }^{8}$ Altri ne furono entusiasti. ${ }^{9}$

L'esempio più significativo di questo rovesciamento riguarda certamente la sua critica agli universali. In essa Ockham non fa appello in maniera diretta all'assunto "non si deve porre una pluralità di cose se non è strettamente necessario" "pluralitas non est ponenda sine necessitate", nè tanto meno si propone di dimostrarlo; egli piuttosto argomenta in maniera logicamente valida contro certe assunzioni ontologiche, cioè che l'universale sia una substantia. L'inaccettabilità di questo assunto risulta chiara dalle conseguenze che ne derivano e che vengono puntigliosamente elencate. Nel caso specifico ciò significa che queste entità non sono solamente non necessarie od inutili, ma gravide di conseguenze assurde ed inaccettabili. Il rasoio non concede alcun vantaggio ad Ockham in questa discussione, poichè le singole dimostrazioni vanno fatte sul terreno degli avversari. Ma ora l'avversario non può più rifugiarsi nella presunta inviolabilità dei propri principi perchè egli stesso ha ammesso una convenzione metodologica che sta a monte di tali principi e che consente una loro critica o una loro reinterpretazione, ogniqualvolta la loro applicazione avvenga in contrasto con l'assunto iniziale.

Ockham non confonde la spiegazione logica con la spiegazione metafisica, ma è convinto che ambedue hanno in comune alcune proprietà relative appunto alla natura della spiegazione stessa. In base a ciò non può essere lecito in metafisica ciò che non è lecito in logica. Proprio per tale esigenza di uniformità il principio logico "tutti gli enunciati contradittori hanno lo stesso tipo di inconsistenza tra di loro" ${ }^{10}$ non può essere valido a livello logico e disatteso a livello metafisico. Quando a livello metafisico si fa appello, ad esempio, alla maggior perfezione di alcuni enti rispetto ad altri, tanto che vi sarebbe una maggior contraddizione tra Deus e nonDeus che tra asinus e non-asinus, ciò non significa per Ockham che si voglia risolvere l'ontologia nelle regole della logica, ma significa voler imporre il rispetto dell'assunto di dare spiegazioni uniformi e semplici della realtà. ${ }^{11}$ Diversamente la filo-

8 Cfr. I. LUTERELLIS, Libellus contra doctrinam Guilelmi de Ockham, edizione a cura di F. HOFFMANN, Die erste Kritik des Ockhamismus durch den Oxforder Kanzlers Johannes Lutterell, Breslau 1941, p. 7, 33-36: "... conceptiones aliquas repperi, que idcirco aures multorum offendunt, quoniam imperitos decipiunt per philosophiam inanem et fallacem, periculosiores iam creduntur in tantum quod, si inter theologos libera licentia coalescant, doctrine sane et catholice obruent veritatem ..."; p. 26, 26- 30: "Quamvis autem ista sint puerilia, quia tamen hec sunt isti in loycalibus principalia fundamenta et per ista non solum doctorum omnium priorum ac etiam philosophorum, sed principia puerorum subvertit ...".

9 In effetti, le dottrine di Ockham ottennero un vasto successo non solo ad Oxford e a Parigi, ma anche in molte altre sedi universitarie. La nascita di un movimento "ockhamista" è documentata a vari livelli; esso poi ha avuto modo di svilupparsi per un lungo periodo. Cfr. F. BOTTN, La scienza degli occamisti: la scienza tardo-medievale dalle ongini del paradigma nominalista alla rivoluzione scientifiCa, Maggioli, Rimini 1982, p. 19-66.

10 Cfr. OCKHAM, In I Sent., d. 2, q. I, p. 15, 6-7; 174, 8-9: "omnia contradictoria habent aequalem repugnantiam inter $\mathrm{se}^{\mathrm{u}}$.

11 Crr. OCKHAM, In I Sent., d. 2, q. VII, p. 248, 8-21: "... non bene dicuntur talia communia: quod eadem res sub una intentione est singularis et sub alia universalis, vel quod secundum tale esse est homo et secundum aliud esse non est homo, sed aliud et multa talia consimilia, puta si res consideretur sic, tunc est hoc, si aliter consideretur vel sub alia consideratione, est aliud; quia tales propositiones accipientes tales determinationes inferunt propositiones absolute sine illis determinationibus. 
sofia rinuncerebbe ad essere una spiegazione della realtà e diventerebbe un racconto estremamente complesso, forse affascinante, della ragione umana sulla perfezione di ogni entità reale. Chiarito ciò, l'argomentazione di Ockham può procedere quasi ad hominem per demolire la posizione sugli universali di Giovanni Duns Scoto.

Un procedimento analogo viene utilizzato per distruggere l'intreccio tomistico tra ente logico ed ente reale, che l'oxoniense considera un gioco di prestigio linguistico non necessario per spiegare la realtà. Utilizzare tali procedimenti significa dare origine a quelle tipiche situazioni, linguistiche ed ontologiche, nei cui meandri i filosofi sfuggono ad ogni critica razionale. Come discutere con chi ammette che la stessa cosa ora può essere universale, ora singolare, che lo stesso individuo ora può essere uomo ora non-uomo, che lo stesso animale può essere un bue, un asino o un leone? Non solo il linguaggio filosofico in tal modo gira a vuoto, ma infrange pure il fiat metodologico accettato e perci finisce per costruire una spiegazione che non rispetta i canoni inizialmente ammessi.

\section{5 - Spiegazione filosofica e critica razionale}

L'intento di Ockham in ambedue i casi è quello di rendere possibile la spiegazione filosofica e quindi la critica razionale. Ora, dare una spiegazione razionale significa fondamentalmente avere la possibilità di scelta tra differenti spiegazioni, più o meno esaustive, in nome di alcuni criteri universalmente condivisi. Ciò comporta produrre delle critiche e delle controspiegazioni su basi comunemente accettate in modo da ridurre, il più possibile, la gratuità e l'arbitrarietà implicite in ogni tipo di scelta. L'ultimo terreno comune, oltre il quale si entra nel regno del soggettivo e del gusto individuale, è costituito appunto da una convenzione metodologica capace di rispecchiare l'esigenza di intersoggettività propria di ogni spiegazione; questo terreno è quello della semplicità e della economicità nella spiegazione razionale.

Ockham, d'altra parte, tanto è esplicito e rigoroso nell'imporre il rispetto dell'assunto metodologico, quanto poi è tollerante sulle differenti posizioni che i filosofi possono assumere nelle loro spiegazioni razionali: "questa opinione avrebbe potuto essere espressa in modo diverso ... Queste opinioni non possono essere facilmente smentite, né sono inaccettabili, né contengono una falsità cosi evidente ... considero accettabile ciascuna di queste tre opinioni, ma lascio al giudizio degli altri stabilire quale di esse sia la più vera...". ${ }^{12}$

In tutto il dibattito sugli universali egli insiste nel sostenere di essersi proposto unicamente di mettere in evidenza l'assurdità linguistica ed ontologica di postulare l'esistenza di entità che risultano essere non necessarie, cioè di sconfiggere la gra-

Aliter enim eadem facilitate possem dicere quod homo secundum unum esse vel sub una consideratione vel intentione est unus asinus et sub alia est unus bos et sub alia est unus leo, quod est absurdum. Et ideo tales sunt nimis improprii modi loquendi et abiciendi ab omni modo loquendi scientifi$\mathrm{con}$.

12 Crr. OCKHAM, In I Sent., d. 2, q. VIII, p. 291, 7; 16-17: "ista opinio posset diversimode poni ... Istae opiniones non possunt faciliter improbari, nec sunt ita improbabiles nec ita evidentem falsitatem continent ... quamlibet istarum trium opinionum reputo probabilem, sed quae earum sit verior relinquo iudicio aliorum". 
tuità linguistica e metafisica: "io sostengo una sola cosa e cioè che nessun universale, a meno che non si tratti di un universale stabilito convenzionalmente, è qualcosa di esistente in qualunque modo fuori dell'anima, ma tutto ciò che è universale che per sua natura può essere predicato di molti esiste solo nella mente o in maniera soggettiva oppure in maniera oggettiva; pertanto nessun universale può essere per sua essenza o definizione una sostanza..."13

Un altro esempio particolarmente significativo di questa metodologia può essere ricavato dal confronto delle dottrine sulla potentia Dei absoluta di Tommaso d'Aquino e di Guglielmo di Ockham. Per molti aspetti le due posizioni sembrano incredibilmente vicine: per ambedue i pensatori medievali la distinzione tra potentia ordinata e potentia absoluta non deve far pensare che in Dio vi siano due potentiae che potrebbero venire reciprocamente in conflitto; per ambedue il limite alla potenza divina è costituito dal rispetto del principio di non contraddizione, per ambedue le cose che Dio non può fare sono piuttosto cose che non possono essere fatte per loro natura. ${ }^{14}$

Ma mentre Tommaso è in grado di elencare una lunga lista di cose che neanche Dio potrebbe fare nemmeno de potentia absoluta, onde evitare il contrasto con la fisica e la metafisica di Aristotele, Ockham si preoccupa di sfuggire alla tendenza di considerare contraddittorio ciò che è semplicemente contrario alle singole dottrine aristoteliche. In particolare, il rispetto del principio di non contraddizione non gli impedisce, come avviene in Tommaso di proporre nuove concezioni in naturalibus.$^{15}$ Mentre Tommaso aristotelicamente afferma che "poichè i principi di alcune scienze l'essenza della cosa stessa, ne consegue che Dio non può fare ciò che è contrario a tali principi", ${ }^{16}$ Ockham non ha dubbi nell'affermare che tali principi formali non possono consentire di fondare rigorosamente, ad esempio, la dottrina dell'unicità dell'universo. La dottrina opposta, la pluralità dei mondi può essere argomentata razionalmente in maniera altrettanto plausibile, pur nel rispetto dei prin-

13 Cfr. OCKHAM, In I Sent., d. 2, q. VIII, p. 291, 17-292, 1 : "Hoc tantum teneo, quod nullum universale, nisi forte sit universale per voluntariam institutionem, est aliquid existens quocumque modo extra animam, sed omne illud quod est universale praedicabile de pluribus ex natura sua est in mente vel subiective vel obiective, et quod nullum universale est de essentia seu quidditate cuiuscumque substantiae".

14 Crr. L. MOONAN, St. Thomas on Divine Power, in Atti del Congresso Internazionale per il VII Centenario di san Tommaso, Roma-Napoli 1974, vol. III, p. 366-407; A. GHISALBERTI, Onnipotenza divina e contingenza del mondo in Guglielmo di Ockham, in Sopra la volta del mondo. Onnipotenza e potenza assoluta di Dio tra Medioevo e età modema, Lubrina, Bergamo 1986, p. 33-56. Ma la recente letteratura su questi argomenti è particolarmente ricca; si veda la bibliografia in Sopra la volta del mondo, curata da E. Randi, p. 175-183.

15 La discussione sulla possibilità di altri mondi è tutta rivolta a rendere ammissibile da un punto di vista epistemologico la pluralità dei mondi, pur senza combattere espressamente i principi della fisica aristotelica che vi si opponevano. A tale scopo Ockham adduce il noto esempio di carattere empirico dei due fuochi, ad Oxford e a Parigi, i quali seguendo le leggi della fisica aristotelica si elevano verso l'alto, ma certamente non arrivano ad occupare lo stesso luogo. Analogamente due terre, appartenenti ad universi distinti, non finirebbero per occupare lo stesso luogo nello spazio, pur mantenendo tutte le proprieta che Aristotele aveva previsto per l'elemento terra (cfr. OCKHAM, Ordinatio, d. 44, p. 657-661).

16 Cfr. TOMMASO D'AQUINO, Summa contra Gentiles, lib. II, cap. 25: "... cum principia quarumdam scientiarum ... sumantur ex solis principiis formalibus rerum ex quibus essentia rei dependet, sequitur quod contraria horum principiorum Deus facere non possit ...". 
cipi formali della scienza. Ancora una volta il sacco della farina aristotelica non è andato disperso, ma è stato rivoltato. Ockham non ha alcun interesse a confutare la posizione aristotelica in proposito, ma il rovesciamento del sacco consente di vedere da che cosa esattamente è tenuta insieme la farina filosofica e scientifica che contiene; ora sappiamo che cosa permette ad Aristotele e ai suoi seguaci di raccogliere una messe cosi' abbondante ed apparentemente cosi' sicura. Nello stesso tempo abbiamo esatta percezione dei costi esplicativi che tale operazione comporta.

\section{6 - Scomporre e ricomporre i pezzi del puzzle filosofico}

Il meccanismo filosofico è cosi' smontato. Ai nostri piedi stanno(i) alcune indicazioni metodologiche sulla natura della spiegazione filosofica, da tutti più o meno consapevolmente accettate, anche se poco rigorosamente rispettate, (ii) i principi filosofici fondamentali e (iii) i principi propri di ciascuna scienza che vengono applicati ai vari ambiti del reale. Possiamo pretendere un rispetto maggiore delle indicazioni di metodo esplicativo, possiamo di conseguenza rivedere l'applicazione dei principi filosofici generali nelle varie scienze e, pur facendo riferimento agli stessi dati da spiegare, possiamo ricostruire il puzzle della spiegazione filosofica e scientifica con un risultato complessivo molto differente. Il nuovo fiat metodologico ha consentito alla critica razionale di penetrare nell'edificio esplicativo e ha permesso di mettere alla prova i vari sostegni, modificandone in tal modo profondamente la mappa complessiva.

Restando fedele all'assunto metodologico di non ammettere l'esistenza di entità che per esperienza o in seguito ad una dimostrazione razionale 0 in forza dell'autorità della rivelazione, Ockham concentra la sua attenzione filosofica sul nesso esplicativo che connette le varie entità. L'abilità del filosofo consiste nello sviluppare una o più strategie razionali capaci di spiegare i dati ammessi, senza moltiplicare i dati stessi arbitrariamente. Il non aver ritenute fondate le entità della metafisica, non comportò per Ockham una aprioristica sfiducia nelle possibilità esplicative della metafisica stessa, ma impose ai metafisici l'impegno di dimostrare razionalmente, cio rispettando la natura della spiegazione razionale, l'esistenza delle entità eventualmente postulate.

Agli occhi di Ockham i filosofi che sulla base di dottrine aristoteliche hanno costruito una metafisica ripiena di enti puramente metafisici hanno commesso un duplice errore: (i) hanno trasformato in entità reali le connessioni puramente razionali proposte da Aristotele e ciò facendo, di conseguenza, (ii) hanno infranto l'assunto metedologico della uniformità e della semplicità della spiegazione.

La filosofia deve ritornare al suo compito tradizionale, che è quello di spiegare razionalmente e non quello di creare un universo di entità metafisiche accanto a quello della realtà ammessa appunto sulla base dell'esperienza, della dimostrazione o della rivelazione. La filosofia, perciò, è principalmente una strategia razionale rivolta alla ricomposizione di un puzzle ora molto più vasto e più ricco rispetto a quello aristotelico, poichè la rivelazione lo ha arricchito di alcuni dati ad Aristotele ignoti, cioè che l'universo è stato creato da Dio, che Dio è onnipotente, ecc. L'introduzione di queste ipotesi teologiche invece di coartare la libertà razionale del filo- 
sofo, la potenziano straordinariamente. Esse diventano lo stimolo per disporre diversamente anche il disegno complessivo delle realtà conosciute con l'esperienza e per rivedere la stessa coerenza interna delle conclusioni proprie della scienza filosofica.

\section{7 - Tutti i modelli esplicativi sono provvisori}

Per Ockham è stato possibile superare Aristotele e la sua scienza, o meglio un certo modo di presentarla, restando aristotelico perchè egli sembra essere consapevole che il modello epistemologico che noi attribuiamo alla ragione umana non può essere definitivo. Pur accettando alcune acquisizioni fondamentali (i principi dei metafisici), non siamo mai autorizzati a ricavarne una unica e fissa immagine dei modi in cui tali principi possono essere applicati alla realtà, poichè il disegno complessivo sfugge sempre alla mente dell'uomo. Di conseguenza la ragione umana esplica le sue potenzialità quando argomenta con fondamento a favore di una determinata immagine della realtà, ma resta consapevole dell'importanza di confrontare tali immagini con altre possibili rappresentazioni. Tutto ciò può venire considerato in parte come un atteggiamento rinunciatario, una metamorfosi che la ragione volontariamente si impone per non soccombere di fronte alle difficoltà teoretiche, ma è prima di tutto la consapevole accettazione del mutare storico delle forme della razionalità stessa.

Anche della filosofia contemporanea si dice che preferisce inseguire la metamorfosi del logos nel post-moderno assumendo l'aspetto di un pensiero debole, rinunciando a tutto ciò che è fondativo. Ma questa presunta debolezza del pensiero non è altro che una consapevole ritirata della razionalità nei propri limiti. L'esigenza di "fondare" è certamente insita nell'ideale del filosofo di ogni tempo. Ma ciò ha spinto molto spesso la ragione umana oltre i suoi confini ed ha comportato non solo improvvise perdite di fiducia (crisi della ragione), ma soprattutto ha condotto a fondare anche ciò che non dovrebbe mai essere fondato, cioè i propri errori, le proprie intolleranze, in una parola gli aspetti irrazionali che si annidano nell'animo e nella mente dell'uomo. 